\title{
Foot ulcers and their association with diabetic Charcot foot complications
}

\author{
Nabilah Abdul Rahman, \\ Aishah Ahmad Fauzi, Tze Yang Chung, \\ Lydia Abdul Latif, Soo Chin Chan
}

\section{Background and objective}

Diabetic Charcot foot (DCF) can cause gross structural deformities of the foot and ankle. The main objective of this study was to identify complications of DCF and its associated factors.

\section{Methods}

This is a retrospective cohort study. Data on medical background, previous DCF treatment and complications were obtained. Multiple logistic regression analysis was performed to measure factors related to various complications of DCF.

\section{Results}

Ninety-eight patient records were retrieved. Of the 83 patients who were still alive, $75.9 \%(n=63)$ had recurrent ulcers, $53.0 \%(n=44)$ had undergone foot surgery and $45.8 \%(n=38)$ had undergone amputation. Patients with a history of recurrent ulcers have the highest predilection to amputation (odds ratio: 8.5 ; $95 \%$ confidence interval: 1.8, 39.1).

\section{Discussion}

In terms of DCF complications, foot ulcers are an independent predictor of recurrent foot ulcers, foot surgery and amputation. Regular foot assessment of patients with DCF to prevent ulcers is strongly recommended.
DIABETIC CHARCOT FOOT (DCF) is a progressive degenerative arthropathy, and its formation is postulated to originate from repeated microtrauma due to the loss of protective sensation as a result of diabetic sensory neuropathy. ${ }^{1,2}$ The presentation of DCF can be acute or chronic. Acute DCF is characterised by unilateral foot swelling with erythema, elevated foot temperature and a bounding peripheral pulse. This is often misdiagnosed as osteomyelitis and cellulitis, especially in the presence of foot ulcers and absence of foot deformities and X-ray abnormalities. Distinguishing between infection and DCF in the acute stage is challenging, especially when availability of investigations is limited in the primary care setting. When a patient with diabetes presents with soft tissue and bone deformity of the foot in addition to loss of protective sensation, a high index of suspicion of DCF should be made to initiate appropriate management. ${ }^{3}$

DCF may cause gross structural deformities of the foot and ankle and subsequent skin ulceration leading to lower limb amputation if it is not managed early with appropriate measures. Several studies have observed multiple complications after DCF for a follow-up period ranging from two to 12 years. ${ }^{4-10}$ Foot ulcers have been reported as one of the most common complications after DCF in most observational studies, occurring in $11-60 \%$ of cases. ${ }^{5,6,8,10}$ The need for surgery and amputation at the DCF site was also observed during followup. ${ }^{4,5,7-10}$ Between four and five years of DCF follow-up, the mortality rate was observed to be $21-33 \% .^{5-7}$ Of 115 patients followed up by Fabrin et al, $43 \%$ had developed recurrent acute Charcot foot within the median of two years of follow-up. ${ }^{8}$ In another study of 301 patients with DCF, $28 \%$ were noted to have impaired mobility as one of their complications over a follow-up period of 2.5 years. $^{9}$

Foot ulcers, lower limb amputation, foot surgery and mortality had been observed in previous studies as complications following DCF. There is still a paucity of literature on DCF complications and its related associated factors in Asia, hence the primary objective of this study. The four main complications explored were recurrent foot ulcers, foot surgery, amputation and mortality.

\section{Methods}

This was a retrospective cohort study. Data were obtained from patients attending the Diabetic Foot and Wound Management Clinic of University Malaya Medical Centre (UMMC) from 2001 to 2014 through the clinic attendance census. Patients without diabetes who presented with Charcot foot were excluded from the study. The study 
was approved by the UMMC Ethics Committee (MECID:201401-0659).

A face-to-face interview was conducted to gather details on patients' sociodemographic statuses. Physical examination was performed to determine each patient's current foot status, followed by a review of the patient's medical records to determine management received. For deceased subjects, only their medical record data were reviewed.

Ninety-eight sets of data for patients with DCF were collected between 2001 and 2014, with a mean follow-up time of 5.11 years. From the total data, 83 patients $(84.7 \%)$ were still alive during the study period. The four main complications identified were foot ulcers, foot surgery, amputation and mortality. Inferential statistical analysis was performed using simple and multiple logistic regressions (SPSS version 21.0) to determine associated factors for all DCF complications. Kaplan-Meier survival analysis was generated to obtain the mean survival time for mortality.

\section{Results}

Descriptive results from the demographic profile showed equal sex distribution, with $48.2 \%$ male patients $(n=40)$ and $51.8 \%$ female patients $(n=43)$. The mean age of the patients was $59.2 \pm 9.2$ years. Table 1 describes the details of demographic profiles.

In terms of medical comorbidities, $86.7 \%(n=72)$ had hypertension, $71.1 \%$ $(\mathrm{n}=59)$ had dyslipidaemia, $39.8 \%(\mathrm{n}=33)$ had ischaemic heart disease and only $21.7 \%(n=18)$ had end-stage renal failure. In addition, $89.1 \%(\mathrm{n}=68)$ had diabetes for $>10$ years and $76 \%(n=63)$ had poorly controlled glycated haemoglobin (HbA1c) with readings of $>48 \mathrm{mmol} / \mathrm{mol}(6.5 \%)$.

Acute and chronic Charcot foot were diagnosed by the attending physician at the Diabetic Foot and Wound Management Clinic, and diagnoses were based on physical examination with radiological investigation (foot X-ray). Table 2 summarises the DCF profile descriptions.

With regard to the main complications in patients with DCF, 75.9\% $(n=63)$ had recurrent ulcers followed by foot surgery and lower-limb amputation
(Table 3). Other secondary outcomes were ambulation and driving status after being diagnosed with DCF. Findings showed that $53.0 \%(\mathrm{n}=44)$ required aid for ambulation and only $32.5 \%(n=27)$ continued to drive.

The majority of patients $(n=63 ; 75.9 \%)$ had recurrent ulcers post-diagnosis of DCF, and $44.4 \%(n=28)$ had more than three episodes of recurrent ulcers. Analysis with confounder adjustments showed that patients with ulcers and DCF had a higher risk of recurrent ulcers (odds ratio [OR] 4.27; 95\% confidence interval [CI]: $1.38,13.21 ; P=0.01)$ than those without ulcers (Table 4 ).

A total of 44 patients (53\%) had foot surgery after DCF. The majority of foot surgeries occurred during the initial year of diagnosis $(n=26 ; 59.1 \%)$. Multiple logistic regression analysis showed that patients with recurrent foot ulcers had a higher risk of foot surgery (OR 3.41; 95\% CI: $1.13,10.32 ; P=0.03$ ) than those without recurrent ulcers when adjusted for other confounding variables (Table 4).

Almost half of the patients with DCF ( $\mathrm{n}=38 ; 45.8 \%$ ) had complications with lower-limb amputation. Most of the amputations $(n=20 ; 52.6 \%)$ occurred during the first two years following diagnosis of DCF. Multiple logistic regression analysis showed that patients with recurrent ulcers had eight times the likelihood of having an amputation (OR 8.47; 95\% CI: 1.83, $39.16 ; P=0.006)$ followed by heart disease (OR 5.23; 95\% CI: 1.42, 19.20; $P=0.01$ ) and chronic DCF (OR 3.91; 95\% CI: 1.12, 12.99: $P=0.03$; Table 4).

The mortality outcome during the follow-up period was $15.3 \%(n=15)$. The majority of the 15 deceased patients were between 51 and 70 years of age $(n=10)$ and had macrovascular and microvascular complications of diabetes. The mean survival time based on Kaplan-Maier survival analysis was $44.8 \pm 5.5$ months (Figure 1 ).

In relation to mobility outcomes, almost $88 \%$ of the patients $(n=73)$ were ambulating without aid prior to being diagnosed with DCF. Patients with foot ulcers at diagnosis had a significant association with aided premorbid ambulation $(P=0.039)$ in comparison to patients without ulcers at diagnosis.

Table 1. Demographic profiles $(n=83)$

\begin{tabular}{llrr}
\hline Demographic & & Frequency (n) & Percentage \\
\hline \multirow{2}{*}{ Sex } & Female & 40 & 48.2 \\
\hline Age (years) & $<50$ & 43 & 51.8 \\
\cline { 2 - 4 } & $51-70$ & 64 & 14.5 \\
\cline { 2 - 4 } & $>70$ & 7 & 77.1 \\
\hline Ethnicity & Malay & 44 & 8.4 \\
\cline { 2 - 4 } & Chinese & 72 & 53.0 \\
\cline { 2 - 4 } & Indian & 47 & 8.4 \\
\hline Education level & Secondary school & 18 & 38.6 \\
\cline { 2 - 4 } & Tertiary education & 16 & 56.6 \\
\hline Occupation & Homemaker/ & 17 & 21.6 \\
\hline & Unemployed & 50 & 19.3 \\
\cline { 2 - 4 } & Desk job & & 20.5 \\
\cline { 2 - 4 } & Non-desk job & 70.2 \\
\hline
\end{tabular}




\section{Discussion}

Complications related to DCF were the result of a complex cascade of diabetes and neuropathy causing distortion of the normal bony architecture of the foot, which can result in the development of rocker bottom foot. ${ }^{2,11}$ The deformity and ankle instability cause ulceration and infection, which subsequently lead to the requirement for foot surgery and amputation. $^{12}$

Recurrent ulcers after DCF were more frequent in the current study (75\%) when compared with previous studies (11-67\%)..$^{6,8,13}$ Patients who presented with an ulcer with DCF were four times more likely to have recurrent foot ulcers later. Foot surgery was performed for $53 \%$ of the patients, which is similar to the percentage reported in other studies. ${ }^{1,6,8}$ Furthermore, other studies noticed a bimodal distribution for surgical intervention during the first year and third to fourth year post-diagnosis due to recurrent ulcers, foot infections and foot deformities. ${ }^{1,6,8}$ Patients with DCF who had recurrent ulcers were more likely to undergo foot surgery (OR 3.413).

Of the patients who had surgery, more than $50 \%$ had wound debridement and incision and drainage related to infective ulcer management. Corrective surgery with arthrodesis was performed in all patients who underwent surgery. Arthrodesis and exostomy are commonly done for unstable and deformed Charcot foot. ${ }^{1}$ A timely surgery, adequate strong fixation and a long non-weight bearing postoperative period of at least $12-18$ weeks are necessary to enhance good outcomes in DCF. ${ }^{13}$ Surgical intervention with reconstruction surgery also needs to be carefully evaluated and justified. ${ }^{1,14}$ Findings of surgical outcomes after DCF indicate that almost half of the patients with DCF presented with unstable joint and foot deformity. In this case, ongoing weight-bearing may lead to uneven pressure distribution at the plantar of the foot, hence creating ulceration and leading to infection that warrants management (eg with debridement and incisional drainage). ${ }^{15}$

This study also showed a higher percentage of lower limb amputation (45.8\%) than other similar studies, which reported figures of between $4.1 \%$ and $23.4 \% .^{4,5,8}$ Patients with recurrent ulcers had a higher risk of having an amputation than patients without recurrent ulcers. ${ }^{16}$ The overall amputation risk for Charcot foot was found to be not significantly different from diabetic foot ulcers. ${ }^{4}$ Nevertheless, the observations

\section{Table 2. Diabetic Charcot foot profiles $(n=83)$}

\begin{tabular}{llrr}
\hline Diabetic Charcot foot information & Frequency (n) & Percentage \\
\hline $\begin{array}{l}\text { Diabetic Charcot foot } \\
\text { during diagnosis }\end{array}$ & Acute & 54 & 65.1 \\
\cline { 2 - 4 } $\begin{array}{l}\text { Current diabetic Charcot } \\
\text { foot status }\end{array}$ & Acute & 29 & 34.9 \\
\cline { 2 - 4 } & Chronic & 5 & 6.0 \\
\cline { 2 - 4 } & Amputated & 75 & 90.4 \\
\hline Ulcer during diagnosis & Present & 3 & 3.6 \\
\hline Current ulcer & Present & 42 & 50.6 \\
\hline Non-surgical treatment & Yes & 72 & 45.8 \\
\cline { 2 - 4 } & No & 11 & 86.7 \\
\hline Removable boot walker & Yes & 23 & 13.3 \\
\hline Modified shoes & Yes & 62 & 31.9 \\
\hline Casting & Yes & 33 & 86.1 \\
\hline Padding & Yes & 10 & 45.8 \\
\hline Foot insole & Yes & 69 & 13.9 \\
\hline
\end{tabular}

Table 3. Complications of diabetic Charcot foot during follow-up* $(n=83)$

\begin{tabular}{llrrr}
\hline Complications & & Frequency (n) & Percentage \\
\hline Recurrent ulcer & Present & Absent & 63 & 75.9 \\
\cline { 2 - 5 } Surgical treatment & Yes & No & 20 & 24.1 \\
\cline { 2 - 5 } & Yes & 34 & 53.0 \\
\hline Arthrodesis & Yes & 44 & 47.0 \\
\hline Exostomy & Yes & 38 & 100.0 \\
\hline Wound debridement & Yes & 18 & 15.9 \\
\hline Incision and drainage & Yes & 38 & 86.4 \\
\hline Amputation status & Yes & 45 & 40.9 \\
\hline Amputation level & Rays & 28 & 45.8 \\
\cline { 2 - 4 } & Transmetatarsal & 1 & 54.2 \\
\cline { 2 - 4 } & Transtibial & 9 & 73.7 \\
\hline
\end{tabular}

*Mean follow-up time of 5.11 years 


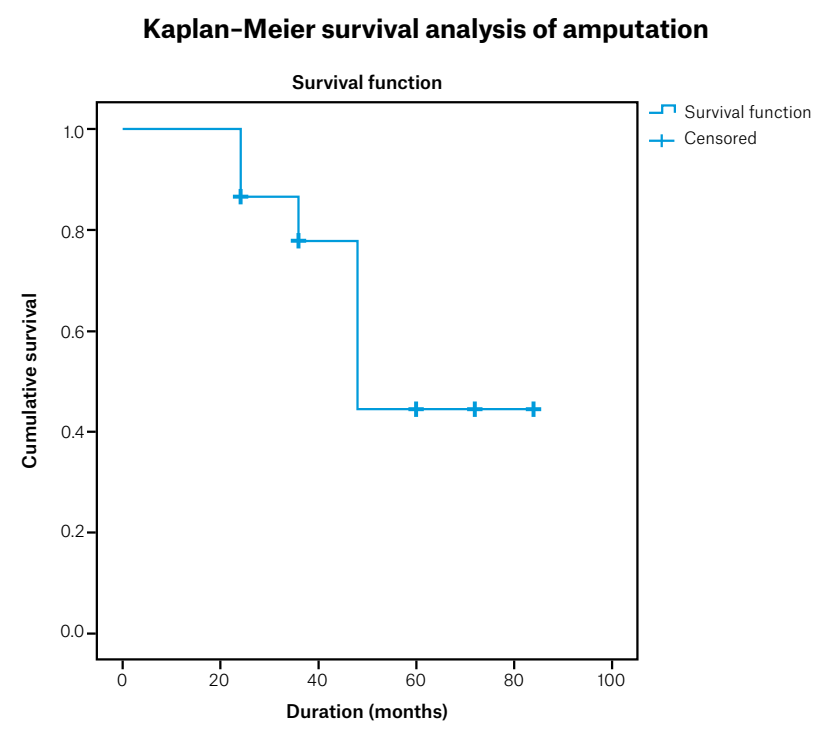

\begin{tabular}{cccc}
\hline & Mean survival time for amputation (months) & \\
\hline Mean estimate & Standard error & \multicolumn{2}{c}{$95 \%$ confidence interval } \\
\cline { 3 - 4 } & & Lower limit & Upper limit \\
\hline 59.806 & 6.921 & 46.240 & 73.372 \\
\hline
\end{tabular}

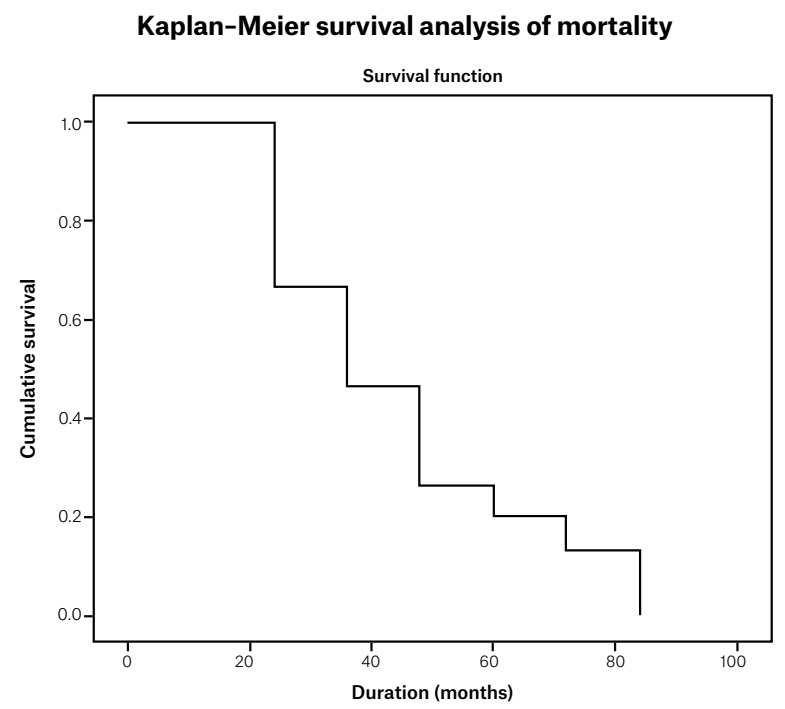

\begin{tabular}{cccc}
\hline & \multicolumn{2}{c}{ Mortality mean survival time (months) } \\
\hline \multirow{2}{*}{ Mean estimate } & Standard error & \multicolumn{2}{c}{$95 \%$ confidence interval } \\
\cline { 3 - 4 } & & Lower limit & Upper limit \\
\hline 44.80 & 5.55 & 33.92 & 55.68 \\
\hline
\end{tabular}

Figure 1. Kaplan-Meier survival analysis graphs for amputation and mortality 


\section{Table 4. Multiple logistic regression analysis on predictors of Charcot foot complications}

\begin{tabular}{|c|c|c|c|c|c|c|}
\hline \multirow[b]{2}{*}{ Outcomes } & \multirow[b]{2}{*}{ Associated factors } & & \multirow[b]{2}{*}{ Odds ratio } & \multicolumn{2}{|c|}{$95 \%$ confidence interval } & \multirow[b]{2}{*}{$P$ value } \\
\hline & & & & Lower limit & Upper limit & \\
\hline Recurrent ulcer & Ulcer during diagnosis & Present & 4.27 & 1.38 & 13.21 & 0.012 \\
\hline Foot surgery & Recurrent ulcers & Present & 3.41 & 1.13 & 10.31 & 0.030 \\
\hline \multirow[t]{3}{*}{ Amputation } & Ischaemic heart disease & Yes & 5.23 & 1.42 & 19.20 & 0.013 \\
\hline & $\begin{array}{l}\text { Stage of Charcot } \\
\text { at diagnosis }\end{array}$ & Chronic & 3.91 & 1.12 & 12.99 & 0.026 \\
\hline & Recurrent ulcers & Present & 8.47 & 1.83 & 39.16 & 0.006 \\
\hline
\end{tabular}

The mortality outcome during the follow-up period was $15.3 \%(n=15)$. Other studies have reported mortality percentages from $3.63 \%$ to $44.7 \% .^{5-7,9,16,20}$ The main cause of death in the current study was medical complications of diabetes. This result is similar to the study by Moulik et al, which indicated that clinical conditions related to cardiovascular origin, such as cardiac arrest and stroke, contributed to the majority of causes of death of patients with DCF. ${ }^{16}$

There are several limitations of this study, especially in obtaining data on the type of treatment done and foot complication status for deceased subjects because of missing data in the medical record. In this study, patients' compliance for non-surgical intervention was not assessed because of its retrospective nature, which may lead to recall bias. Nevertheless, comparison of outcomes between patients on total contact casting and with removable pressure offloading devices might be useful in future prospective studies where missing data and recall bias can be avoided. Another limitation relates to peripheral vascular disease, which is one of the common comorbidities among patients with diabetes who have foot concerns but was not captured during data recruitment.

\section{Conclusion}

Foot ulceration was identified as the main factor associated with DCF complications, which include recurrent ulcers, amputation, foot surgery and declining ambulation status. Prevention of ulceration is of utmost importance in reducing the occurrence of DCF complications. Early recognition at the primary care level and active management of DCF may reduce the risk of developing further complications.

\section{Implications for general practice}

- This study focused on DCF, which is a debilitating complication of diabetes.

- Data presented here were from a tertiary care and referral centre in Malaysia.

- Understanding of the complications and predictors of DCF can be applied in clinical services to improve management of DCF and prevent its complications.

- The presence of foot ulcers was the factor most associated with DCF complications.

- Regular assessment of DCF is a core prevention strategy for reducing the risk of DCF complications.

\section{Authors}

Nabilah Abdul Rahman MBBS (IIUM), MRehab Med (UM), Rehabilitation Physician, Ministry of Health, Rehabilitation Medicine, Hospital Sultanah Bahiyah, Alor Setar, Kedah, Malaysia

Aishah Ahmad Fauzi MBBS, MRehab Med Mal, Senior Lecturer and Consultant Rehabilitation Physician, Department of Rehabilitation Medicine, Faculty of Medicine, University of Malaya, Kuala Lumpur, Malaysia.aishahaf@ummc.edu.my

Tze Yang Chung MBBS, MRehab Med Mal, Senior Lecturer and Consultant Rehabilitation Physician, Department of Rehabilitation Medicine, Faculty of Medicine, University of Malaya, Kuala Lumpur, Malaysia Lydia Abdul Latif MBBS, MRehab Med Mal, Professor and Rehabilitation Physician, Department of Rehabilitation Medicine, Faculty of Medicine, University of Malaya, Kuala Lumpur, Malaysia
Soo Chin Chan MBBS, IMU, MRehab Med Mal, Lecturer and Rehabilitation Physician, Department of Rehabilitation Medicine, Faculty of Medicine, University of Malaya, Kuala Lumpur, Malaysia Competing interests: None.

Funding: None.

Provenance and peer review: Not commissioned, externally peer reviewed.

\section{Acknowledgement}

The authors thank the staff of the Diabetic Foot Care and Wound Management Clinic of the University Malaya Medical Centre for their assistance in this study.

\section{References}

1. Wukich DK, Sung W. Charcot arthropathy of the foot and ankle: Modern concepts and management review. J Diabetes Complications 2009;23(6):409-26. doi: 10.1016/j. jdiacomp.2008.09.004

2. Gupta PPK, Mohan V. Charcot foot - An update. J Assoc Physicians India 2003;51:367-72.

3. Ahmad Fauzi A, Chung TY. Bilateral diabetic Charcot foot. Aust Fam Physician 2013;42(1):55-56.

4. Sohn MW, Stuck RM, Pinzur M, Lee TA, Budiman-Mak E. Lower-extremity amputation risk after Charcot arthropathy and diabetic foot ulcer. Diabetes Care 2010;33(1):98-100. doi: 10.2337/dc09-1497.

5. Pakarinen TK, Laine HJ, Mäenpää $H$, Mattila $P$, Lahtela J. Long-term outcome and quality of life in patients with Charcot foot. Foot Ankle Surg 2009;15(4):187-91. doi: 10.1016/j.fas.2009.02.005.

6. Leung HB, Ho YC, Wong WC. Charcot foot in a Hong Kong Chinese diabetic population. Hong Kong Med J 2009;15(3):191-95.

7. Gazis A, Pound N, Macfarlane R, Treece K, Game F, Jeffcoate W. Mortality in patients with diabetic neuropathic osteoarthropathy (Charcot foot). Diabet Med 2004;21(11):1243-46. doi: 10.1111/j.1464-5491.2004.01215.x.

8. Fabrin J, Larsen K, Holstein PE. Longterm follow-up in diabetic Charcot feet with spontaneous onset. Diabetes Care 2000;23(6):796-800. doi: 10.2337/ diacare.23.6.796.

9. Sinacore DR, Withrington NC. Recognition and management of acute neuropathic (Charcot) arthropathies of the foot and ankle. J Orthop Sports Phys Ther 1999;29(12):736-46. doi: 10.2519/jospt.1999.29.12.736. 
10. Armstrong DG, Todd WF, Lavery LA, Harkless LB, Bushman TR. The natural history of acute Charcot's arthropathy in a diabetic foot specialty clinic. Diabet Med 1997;14(5):357-63. doi: 10.1002/ (SICI)1096-9136(199705)14:5<357::AIDDIA341>3.0.CO;2-8.

11. Rogers LC, Frykberg RG, Armstrong DG, et al. The Charcot foot in diabetes. Diabetes Care 2011;34(9):2123-29. doi: 10.2337/dc11-0844.

12. Frykberg RG, Belczyk R. Epidemiology of the Charcot foot. Clin Podiatr Med Surg 2008;25(1):17-28. doi: 10.1016/j.cpm.2007.10.001.

13. Pakarinen TK, Laine HJ, Honkonen SE, Peltonen J, Oksala H, Lahtela J. Charcot arthropathy of the diabetic foot. Current concepts and review of 36 cases. Scand J Surg 2002;91(2):195-201. doi: 10.1177/145749690209100212.

14. Pinzur MS, Evans A. Health-related quality of life in patients with Charcot foot. Am J Orthop (Belle Mead NJ) 2003;32(10):492-96.

15. Game FL. Osteomyelitis in the diabetic foot: Diagnosis and management. Med Clin North Am 2013;97(5):947-56. doi: 10.1016/j. mcna.2013.03.010.

16. Moulik PK, Mtonga R, Gill GV. Amputation and mortality in new-onset diabetic foot ulcers stratified by etiology. Diabetes Care 2003;26(2):491-94. doi: 10.2337/diacare.26.2.491.
17. Sämann A, Pofahl S, Lehmann T, et al. Diabetic nephropathy but not $\mathrm{HbA1c}$ is predictive for frequent complications of Charcot feet - Longterm follow-up of 164 consecutive patients with 195 acute Charcot feet. Exp Clin Endocrinol Diabetes 2012;120(6):335-39. doi: 10.1055/s0031-1299705.

18. Ahmad Fauzi A, Chung TY, Latif LA. Risk factors of diabetic Charcot Foot arthropathy: A case-control study at a Malaysian tertiary care centre. Singapore Med J 2016;57(4):198-203. doi: 10.11622/smedj.2016074.

19. Renner N, Wirth SH, Osterhoff G, Böni T, Berli M. Outcome after protected full weightbearing treatment in an orthopedic device in diabetic neuropathic arthropathy (Charcot arthropathy): A comparison of unilaterally and bilaterally affected patients. BMC Musculoskelet Disord 2016;17(1):504. doi: 10.1186/s12891-016-1357-4.

20. Sohn MW, Lee TA, Stuck RM, Frykberg RG, Budiman-Mak E. Mortality risk of Charcot arthropathy compared with that of diabetic foot ulcer and diabetes alone. Diabetes Care 2009;32(5):816-21. doi: 10.2337/dc08-1695. 